\title{
Discourse Analysis and Faculty Development in Economics: An Italian Case-Study
}

\author{
Luigina Mortari ${ }^{1}$, Fedra Alessandra Pizzato ${ }^{2}$, Roberta Silva $^{3}$, Alessia Bevilacqua $^{4}$ \\ University of Verona (Italy)
}

\begin{abstract}
The technological innovation ignited by the big data has blazed new trails in the world of Higher Education. Nowadays, technology allows us to systematically record entire courses gathering intentional and unintentional words, interactions, and reactions. Data mining opens up new opportunities for the analysis of university teaching, which enable us to spot novel ambits of action for faculty development and for the improvement of the students' learning experience (see es. Lane, 2014).

The research project presented in this paper exploits these opportunities. I discuss a case study of innovation in economics teaching. We have carried out a discourse analysis of dozens of hours of recorded lectures (MA level) delivered at the School of the Economic Sciences of the University of Verona. The aim of our analysis is to highlight the discursive structure of the lectures as well as the teacher-student interaction, to evaluate the efficacy of courses and to suggest further improvements of the teaching method and the faculty development.
\end{abstract}

Keywords: Qualitative Research, Coding, Higher Education, Teaching Methods in Economics, Teaching Development. 